\title{
Preschool period development of implicit and explicit remembering
}

\author{
JULIA L. GREENBAUM \\ University of Toronto, Toronto, Ontario, Canada \\ and \\ PETER GRAF \\ University of British Columbia, Vancouver, British Columbia, Canada
}

\begin{abstract}
A 3-year-old child may spontaneously recall and talk about a recent event, such as the visit of a friend, even though unable to remember the same event when asked specific questions. This phenomenon illustrates the difference between implicit and explicit remembering. In the present study, we presented 3-, 4-, and 5-year-olds with pictures of common objects; then we assessed implicit remembering with a word-production test, and explicit remembering with a recall test. Performance on the latter test showed the expected increase across age groups. In contrast, wordproduction performance showed comparable memory benefits across age groups. This pattern of findings mirrors age changes in memory performance that occur in late adulthood and in patients with anterograde memory deficits.
\end{abstract}

A 3-year-old child may spontaneously recall and talk about a recent event, such as the visit of a friend, even though unable to remember the same event when asked specific questions about it. This phenomenon illustrates the difference between implicit and explicit remembering (Graf \& Schacter, 1985). Implicit and explicit remembering are similar in that both involve the recollection/ evocation of previous events and experiences; the critical difference between them is that explicit remembering is initiated and guided by a conscious intent to remember, whereas implicit remembering is triggered and guided by external and internal cues that occur in the course of other ongoing activities (see Graf \& Schacter, 1989; Istomina, 1975; J. M. Mandler, 1984).

The capacity for explicit remembering develops after the capacity for implicit remembering. This idea was suggested by Istomina (1975; see also J. M. Mandler, 1984; Piaget, 1973), who maintained that during infancy and the preschool period, "memory processes [encoding/ retrieval operations] are not independent processes, but are integrated into some other activity and are involuntary. Later ... these processes are transformed into specific, internal acts.... [Explicit remembering is made possible by] the differentiation of a specific type of act, the purpose of which is to remember or recall something from among the child's overall activity" (Istomina, 1975, pp. 7-8). In this view, the same basic processes underlie

This research was supported by Grants from the Natural Sciences and Engineering Research Council of Canada and the McLuhan Center for Culture and Technology. We thank the staff, children, and parents of the day-care centers where we conducted the study. Address requests for reprints to Peter Graf, Department of Psychology, University of British Columbia, Vancouver, BC V6T 1Y7, Canada. implicit and explicit remembering, but they are engaged and controlled in a different manner. During the early preschool years, these processes are embedded in other activities, such as playing games, and their use is initiated and guided by the contents and structures of those activities. In contrast, in the later preschool years, explicit remembering is enabled by the development of a new capacity for formulating conscious plans (mental models) for initiating and guiding the processes required for memory retrieval.

Elsewhere we have explained the difference between implicit and explicit remembering in adult subjects in terms of two memory-organizing effects-unitization and grouping (Graf \& Schacter, 1989; see also Graf \& G. Mandler, 1984). Unitizing occurs when previously separate stimulus components become represented as a single unit; it is thought to result from perceiving or conceiving coherence or structure among the components of a stimulus item (see Graf \& Schacter, 1989). The advantage of this type of processing comes from the tendency of a unitized representation to become reactivated or redintegrated as a whole when only some of its parts are subsequently reprocessed (e.g., Horowitz \& Prytulak, 1969; G. Mandler, 1980). We assume that some of the processes required for unitization and redintegration (especially those described by Gestalt laws, such as the law of proximity or the law of good continuation) are functional and influence remembering even during the early preschool period because they are enabled by the organization of the perceptual system and by the structure of ongoing activities, as well as by the nature/form of items on tests that tap implicit remembering. Therefore, we believe that even young preschoolers are capable of implicit remembering in test situations that are sufficiently structured to 
initiate and guide the redintegration of unitized representations (see also Moscovitch, 1985).

Grouping is the process through which unitized representations become assembled into a set that defines them as members of the same collection. We assume that constructing this set requires elaborative processing-that is, it requires going beyond the to-be-remembered (TBR) targets to conceive associations among them, as well as among targets, prior knowledge, and many aspects of the experimental situation (see Graf \& Schacter, 1989). Grouping facilitates explicit remembering, because typical instructions for recall and recognition tests specify TBR targets in terms of group-defining features (e.g., remember the words from the cards that I showed you earlier) and subjects orient their responding according to these specifications. As a result, associations among encoded representations provide the routes for accessing and retrieving TBR targets. We share the widespread view that young preschoolers do not have the capacity to initiate and guide the elaborative processing that produces grouping of representations and thereby enables explicit remembering.

To examine the development of the unitizing and grouping processes, we assessed preschoolers' performances on two different tasks, one that clearly taps the capacity for explicit remembering, and one that requires only the capacity for implicit remembering. We presented 3-, 4-, and 5-year-olds with pictures of common objects and then assessed explicit remembering with a recall test. To assess implicit remembering, we adapted a word-production task from recent studies with adult subjects (e.g., see Graf, Shimamura, \& Squire, 1985). For this task, the experimenter tells a brief story about going to some place, such as a zoo or a restaurant, and then asks the child to name what is typically found there. This task does not require explicit remembering of recently presented materials, but it provides an opportunity for implicit remembering, which is demonstrated if recently seen pictures are named more often than in the absence of their presentation (i.e., in a baseline condition). The memory advantage for recently seen items is generally known as priming (Cramer, 1966). We expected no age-related increase in priming on this word-production task, on the assumptions (1) that it provides a relatively pure measure of implicit remembering, and (2) that preschool memory development is due primarily to the acquisition of a processing capacity that mediates grouping of representations and thereby enables explicit remembering. Alternatively, on the assumption that the unitizing processes keep developing alongside the grouping processes, we expected age-related increases on both test types.

\section{METHOD}

\section{Subjects}

The subjects were 36 children from Toronto area preschools: 12 3-year-olds (mean $=3: 5$ years, range $=2: 11$ to 3:11 years), 12 4-yearolds (mean $=4: 5$ years, range $=4: 0$ to $4: 10$ years), and 12 5-yearolds (mean $=5: 5$ years, range $=5: 0$ to $6: 4$ years).
Table 1

Target Words Corresponding to the Pictured Items That Were Presented for Study

\begin{tabular}{llll}
\hline tree & zebra & chair & pot \\
climber & camel & picture & fridge \\
merry-go-round & polar bear & french fries & stove/oven \\
flowers & fish & drinks & eggs \\
fountain & kangaroo & hamburger & bowl \\
bench & bunny & ice cream & bread \\
\hline
\end{tabular}

\section{Materials}

The materials were 27 line drawings of common objects photocopied from different children's books. ${ }^{1}$ Twenty-four pictures were used to form four sets of six targets, and 3 pictures were used for illustrating and practicing the experimental instructions and tasks. Each picture was shown on a $10 \times 15 \mathrm{~cm}$ index card. The names of the target pictures are listed in Table 1.

The six items in each target set were all from the same category; they were obtained from a pilot study in which preschoolers were asked to name items that can be seen in different places-specifically, in a zoo, park, restaurant, and kitchen. Even 3-year-olds are familiar with many items from each of these categories. The six items selected as targets appeared with intermediate frequencies among the items named by the pilot subjects.

For each set of target pictures, we also composed a short story, five to six sentences long. An example appears in Figure 1. The stories were used to instruct subjects on the word-production task. Each story began with the statement "Now I'm going to tell you a story about a little boy/girl your age named...." The story was designed to create a scenario that led the protagonist to a particular place, such as the zoo, and it ended with the following question: "What do you think [name] saw at/in the [place]? Tell me everything you think he/she might have seen."

\section{Procedure}

Each child was tested individually at the daycare center in a quiet area away from ongoing activities. They were invited to play a memory game with the experimenter. The general procedure involved two study/test trials. To begin each trial, the child was told, "I'm going to show you some pictures, and all I want you to do is tell me what they are, and try to remember them because I'm going to ask you about them later." The task was illustrated with the practice items, and then the 6 pictures from one of the target sets were presented at a pace that allowed subjects to name each (from 3-5 sec per picture).

Immediately after study, the first of two category-production tasks was given. The experimenter instructed the child by telling a brief story (see the Materials section) and then asked him/her to name items from one of the target categories (e.g., things that can be seen in a zoo). The subjects were prompted to name more items until at least six different ones were produced or until they said they could not think of any more. Then a second story was presented, and the subjects were again asked

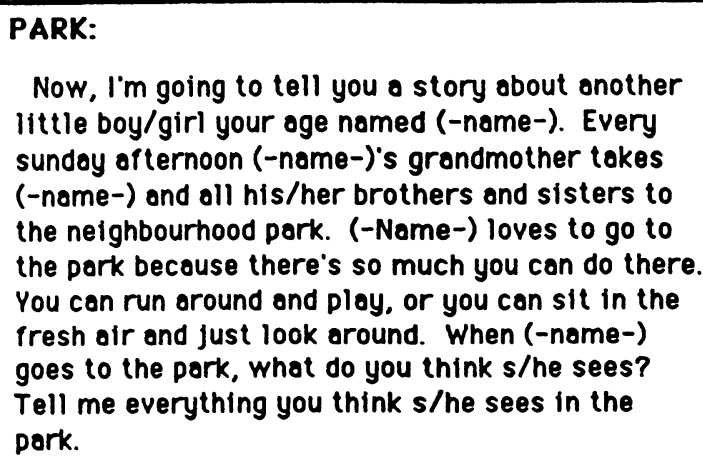

Figure 1. One of the stories used to instruct and guide subjects on the word-production task. 
to name six relevant items. The second story was always used to elicit naming of items related to the studied target set, whereas the first story concerned a category from which no pictures had been studied. Thus, the second story provided a measure of naming of items primed by the previously presented pictures (i.e., the experimental condition), whereas the first story provided a baseline measure of target naming in the absence of prior picture presentation. The performance difference between these conditions provides an index of implicit remembering or priming.

At the end of each study/test trial, the children were tested for explicit remembering of the studied pictures. The experimenter placed the studied pictures face down in front of the child and said, "These are the pictures that I showed you earlier, can you remember and tell me what they were?' 'The children were asked to remember as many pictures as possible. Recall was terminated when a child said he/she could not remember any more. This general procedure was used for both study/test trials. Across subjects, each of the four different target sets was used equally often on each trial, and each was used equally often in the experimental and control conditions.

\section{RESULTS}

The critical results concern the recall of target pictures, and the production of picture names when they were presented for study (in experimental conditions) versus when they were not presented for study (in baseline conditions).

\section{Word Production}

A preliminary analysis of the word-production test results showed that across age groups, there was an increase in the total number of items produced per target set (means $=4.50,5.68$, and 5.94, respectively, for 3-, 4-, and 5-year-olds). This is consistent with other findings of age-related increases in word production (e.g., Nelson, 1974). In view of this increase, we scored test performance in two different ways: first, as a proportion of target words out of six (the size of the study sets), and second, as a proportion of targets out of the total number of words produced per category by each child. Both scoring methods yielded similar patterns of results, and thus only the findings from the former are presented.

Table 2 shows mean performance on the word-production test. The means indicate that all subjects named consistently more targets in the experimental condition (i.e., after seeing target pictures) than in the baseline condition, thereby providing evidence for implicit remembering or priming. More importantly, the means show that the amount of priming was comparable across age groups. This observation is supported by an ANOVA that showed a significant main effect for conditions [experimental vs. baseline; $F(1,33)=26.93, p<.01]$, but no evidence of an interaction with age groups $(F=0.43)$. There was a marginal main effect for groups $[F(2,33)=2.9$,

Table 2

Mean Word-Production Test Performance by 3-, 4-, and 5-Year-Olds in the Experimental and Baseline Conditions

\begin{tabular}{lccc}
\hline & \multicolumn{3}{c}{ Age Group } \\
\cline { 2 - 4 } & 3 years & 4 years & 5 years \\
\hline Experimental condition & $22 \%$ & $23 \%$ & $30 \%$ \\
Baseline condition & $8 \%$ & $13 \%$ & $13 \%$ \\
\hline
\end{tabular}

$p<.07$ ], reflecting the overall age-related increase in word production.

\section{Cued Recall}

Overall cued recall performance was $17 \%, 32 \%$, and 47\%, respectively, for 3-, 4-, and 5-year-olds. An ANOVA of these means showed a significant main effect for age groups $[F(2,32)=9.9, p<.01]$. (Note: One 3-year-old did not finish the recall test and was not included in the analysis.) No other effects approached significance.

\section{DISCUSSION}

These findings illuminate the development of memory during the preschool period. The age-related increase in recall performance replicates well-established results in the literature. The critical new finding is the pattern of priming on the word-production test. Priming is a measure of the influence of prior study on implicit remembering. The finding that age had no influence on implicit remembering indicates that selective aspects of memory are fully functional even in the early preschool years.

To understand why implicit remembering becomes functional before explicit remembering, we focus on the different processing demands of the recall test and of the word-production test. A recall test requires subjects to translate and form into a retrieval plan the experimenter's instructions to recollect the studied items, to remember this plan, and to search memory according to it. An improvement in any of these strategic aspects of remembering will influence recall performance. However, because children do not acquire and use high-level strategies for remembering until later on in their development, we argue that the observed increase in recall performance reflects an additional, lower level processing strategy that produces grouping of representations. As outlined in the introduction, grouping is achieved through processing the study list items in relation to each other and in relation to the experimental situation (Graf \& Schacter, 1989). Previous research has demonstrated that preschoolers do not spontaneously engage in processing that promotes grouping; nevertheless, their memory performance improves when this type of processing is enabled by means of related study materials or by appropriate study activities (e.g., Goldberg, Perlmutter, \& Meyers, 1974; Liberty \& Ornstein, 1973; Perlmutter \& Meyers, 1979). In the present experiment, each study list was composed of items belonging to a single category. We suggest that these materials elicited some degree of grouping of items at least in the older children, thereby facilitating their subsequent recall performance.

The focus of the present experiment was on implicit remembering as indexed by priming on the word-production test. In contrast to a recall test, a word-production test does not require subjects to formulate a plan for memory retrieval. Instead, the retrieval plan is provided by the test instructions-in the present instance, by the story we used to elicit the naming of items from each category. The finding that word-production performance was comparable across age groups indicates that even the youngest preschoolers were able to follow the production test instructions. However, the ability to follow instructions was required for performance in both the baseline and experimental conditions, and thus, it alone does not explain priming effects. As outlined in the introduction, we assume that priming requires that processing an item during the study trial increases the unitization among its features, which in turn facilitates the redintegration of the same item during testing (see Graf \& Schacter, 1989; also G. Mandler, 1980; Graf \& G. Mandler, 1984). Therefore, the present finding of comparable priming effects across age groups suggests, first, that unitization of representations is mediated by basic perceptual and cognitive processes that are functional even during the early preschool years, and second, that access to these representations was successful because it was guided by means of a familiar, meaningful story.

The combined findings from the present study are crucial for completing a pattern of results emerging from a large number of recent ex- 
periments that have compared explicit and implicit remembering across a wide range of conditions and in various subject populations (for a review, see Schacter, 1987). To illustrate: Experiments with young and older adults have shown that whereas explicit remembering performance declines in later life, implicit remembering remains the same (e.g., see Graf \& Schacter, 1985; Light \& Singh, 1987). Similar performance dissociations between explicit and implicit remembering have been observed in studies in which patients with memory deficits caused by disease or injury to the brain have been examined; despite being severely impaired on recall and recognition tests, amnesic patients can show entirely normal performance on priming tests (e.g., see Graf, Squire, \& G. Mandler, 1984). In general, the results from previous studies show that implicit remembering can function independently of explicit remembering; they suggest that implicit remembering is mediated by processes that show little change with age and that can survive damage to the brain, whereas explicit remembering depends on processes that are lost first in late adulthood, and that are most likely to become impaired by injury to the brain. Therefore, the present finding that implicit remembering can be fully functional during the early preschool years even when a child has only minimal capacities for explicit remembering raises the possibility that memory changes in late adulthood and in amnesia reflect a regression to a developmentally earlier mode of cognitive functioning. Ongoing research is being done to examine this possibility. Whatever the outcome, the present findings emphasize that a complete picture of implicit and explicit remembering must be able to accommodate memory changes occurring across the whole lifespan.

\section{REFERENCES}

Battig, W. F., \& Montague, W. E. (1969). Category norms of verbal items in 56 categories: A replication and extension of the Connecticut Category Norms. Journal of Experimental Psychology Monographs, 80(3, Part 2).

Cramer, P. (1966). Mediated priming of associative responses: The effect of time lapse and interpolated activity. Journal of Verbal Learning \& Verbal Behavior, 5, 163-166.

Goldberg, S., Perlmutter, M., \& Myers, N. (1974). Recall of related and unrelated lists by 2-year-olds. Journal of Experimental Child Psychology, 18, 1-8.

GRAF, P., \& MANDleR, G. (1984). Activation makes words more accessible but necessarily more retrievable. Journal of Verbal Learning \& Verbal Behavior, 23, 553-568.

GraF, P., \& Schacter, D. L. (1985). Implicit and explicit memory for new associations in normal and amnesic subjects. Journal of Experimental Psychology: Learning, Memory, \& Cognition, 11, 501-518.

GRAF, P., \& SCHACTER, D. L. (1989). Unitization and grouping medi- ate dissociations in memory for new associations. Journal of Experimental Psychology: Learning, Memory, \& Cognition, 15, 930-940.

Graf, P., Shimamura, A. P., \& SQuire, L. R. (1985). Priming across modalities and priming across category levels: Extending the domain of preserved function in amnesia. Journal of Experimental Psychology: Learning, Memory, \& Cognition, 11, 386-396.

Graf, P., SQuire, L. R., \& Mandler, G. (1984). The information that amnesic patients do not forget. Journal of Experimental Psychology: Learning, Memory, \& Cognition, 10, 164-178.

HoRowitZ, L. M., \& PRYTULAK, L. S. (1969). Redintegrative memory. Psychological Review, 84, 519-531.

Istomina, Z. M. (1975). The development of voluntary memory in preschool-age children. Soviet Psychology, 13, 5-64.

LiberTy, C., \& OrNSTEIN, P. A. (1973). Age differences in organization and recall: The effects of training in categorization. Journal of Experimental Child Psychology, 15, 169-186.

Light, L. L., \& SiNGH, A. (1987). Implicit and explicit memory in young and older adults. Journal of Experimental Psychology: Learning, Memory, \& Cognition, 13, 531-541.

MANDLER, G. (1980). Recognizing: The judgment of previous occurrence. Psychological Review, 87, 252-271.

MANDLER, J. M. (1984). Representation and recall in infancy. In M. Moscovitch (Ed.), The transition from infant to child memory (pp. 75-101). New York: Plenum.

Moscovitch, M. (1985). Memory from infancy to old age: Implications for theories of normal and pathological memory. Annals of the New York Academy of Sciences, 444, 78-96.

Nelson, K. (1974). Variations in children's concepts by age and category. Child Development, 45, 577-584.

Perlmutter, M., \& Myers, N. A. (1979). Development of recall in 2- to 4-year-old children. Developmental Psychology, 15, 73-83.

Piaget, J. (1973). Memory and intelligence. New York: Basic Books. SCHACTER, D. L. (1987). Implicit memory: History and current status. Journal of Experimental Psychology: Learning, Memory, \& Cognition, 13, 501-518.

\section{NOTE}

1. The study included 12 additional drawings corresponding to items from two categories listed in the adult norms of Battig and Montague (1966). Implicit and explicit remembering of these items was tested in a separate study/test trial. The results were ambiguous but suggested interesting similarities with and differences from those reported in this paper.

(Manuscript received April 24, 1989.) 